The University of San Francisco

USF Scholarship: a digital repository @ Gleeson Library |

Geschke Center

Psychology

College of Arts and Sciences

2011

\title{
Cultural Norms Shaping Research Group Interviews with Chinese American Immigrants
}

Christine ML Kwan

Kevin M. Chun

University of San Francisco, chunk@usfca.edu

Catherine A. Chesla

Follow this and additional works at: http://repository.usfca.edu/psyc

Part of the Psychology Commons

\section{Recommended Citation}

Kwan, Christine M. L., Kevin M. Chun, and Catherine A. Chesla. "Cultural Norms Shaping Research Group Interviews with Chinese American Immigrants." Asian American journal of psychology 2.2 (2011): 115-127. http://dx.doi.org/10.1037/a0024184

This Article is brought to you for free and open access by the College of Arts and Sciences at USF Scholarship: a digital repository @ Gleeson Library | Geschke Center. It has been accepted for inclusion in Psychology by an authorized administrator of USF Scholarship: a digital repository@ Gleeson

Library | Geschke Center. For more information, please contact repository@usfca.edu. 
Copyright 2011 American Psychological Association.

This article may not exactly replicate the authoritative document published in the APA journal. It is not the copy of record.

Authoritative document: http://dx.doi.org/10.1037/a0024184 
Published in final edited form as:

Asian Am J Psychol. 2011 June ; 2(2): 115-127. doi:10.1037/a0024184.

\title{
Cultural Norms Shaping Research Group Interviews with Chinese American Immigrants
}

\author{
Christine M. L. Kwan, \\ University of California, San Francisco \\ Kevin M. Chun, and \\ University of San Francisco \\ Catherine A. Chesla \\ University of California, San Francisco
}

\begin{abstract}
Practical knowledge on how to tailor research methods for Asian Americans is relatively scarce despite the rapid population growth of this ethnic group and the ongoing calls for greater cultural competence among researchers. Based on a 4-year qualitative study of family and cultural issues in diabetes management among Chinese American immigrants, this article presents data-based analyses of culturally nuanced group interview processes, and recommendations for conducting culturally appropriate group interviews. Group interview processes were prominently shaped by 4 cultural norms: sensitivity to social hierarchy, monitoring public display of strong emotions, face concerns, and emphasis on group harmony. Strategies for facilitating open and dynamic group interviews in the presence of these norms were identified. Skillful facilitation of group processes, either by accommodating or diffusing norms, was required to promote participant rapport and encourage disclosure of everyday experience.
\end{abstract}

\section{Keywords}

cultural norms; Asian American; Chinese American; qualitative; methodology

The purpose of this study is to present data-based analyses of research group interview processes as shaped by cultural norms and to examine strategies for conducting culturally appropriate interviews vis-à-vis cultural norms. In qualitative inquiry, regardless of the substantive topic under investigation, attention to cultural influences on interviewing processes and other aspects of data collection is crucial for obtaining quality data from participants. This study addresses this issue in the context of group interviews with Chinese American immigrants living with the chronic illness of type 2 diabetes.

Asian Americans are among the fastest growing immigrant groups in the U.S. Constituting $4.2 \%$ (11.9 million people) of the total U.S. population, the Asian American population grew by $48 \%$ between 1990 and 2000, outpacing the overall population growth rate of $13 \%$ (U.S. Census Bureau, 2001). This growth has spawned considerable research on culturally appropriate therapy techniques for treating Asian Americans (Lee, 1997; McGoldrick, Giordano, \& Garcia-Preto, 2005; D. W. Sue \& Sue, 2003). Assuming psychotherapy and qualitative research share a common goal of seeking a full, accurate and rich understanding of clients' or participants' everyday experiences, including their emotions and motivations as well as beliefs and practices, it is imperative that qualitative researchers be culturally competent in handling research processes. However, practical recommendations and guidelines for conducting culturally appropriate research with Asian Americans are relatively scarce. The exceptions are found in Asian American and community psychology 
literatures. Iwamasa and Sorocco (2002) described culturally appropriate research design for studying Asian American older adults. They discussed the importance of building rapport with participants, incorporating participants' input when selecting research questions and formats, and attending to Asian values that might impact researcher-participant interactions. Individual interviews and focus groups were considered to be particularly effective methods because they allowed participants to express themselves in their own ways and communicated respect for participants' opinions. Based on mental health research in a community health facility for Asian Americans, Chen et al. (2005) described ways to collaborate with facility staff, negotiate cultural barriers to community participation, and handle enrollment, refusals, informed consent and incentives in ways that were consonant with participants' cultural explanatory models. Chun et al. (2007) presented ways to handle cultural issues in community-based investigations, including building culturally competent research teams, entering a community, recruiting participants, and collecting and analyzing data.

Overall, the literature suggests research with Asian Americans requires cultural adaptation in research design and implementation, particularly to enhance recruitment, engagement and retention. Missing are examinations of cultural issues specific to group interviews, sometimes called focus groups. It is unclear how group dynamics in group interviews are shaped by cultural norms and what methodological shifts are required as a result. This gap needs to be addressed for two reasons. First, group interviews are an important method of data collection in community research with diverse ethnic groups including Asian Americans, particularly in qualitative inquiry (Okazaki, Lee, \& Sue, 2007; Uba, 2002). When facilitated effectively, group interviews provide a safe place for participants to express and exchange their unique reflections and narratives. Second, cultural norms have been shown to affect Asian and Asian American social interactions in clinical (Lee \& Mock, 2005) and naturalistic settings (Gabrenya \& Hwang, 1996; Leung, 1996). Cultural norms likely affect social interactions in research group interviews as well. Regardless of the topic under investigation, individuals in research group interviews may present themselves and respond to others' comments according to cultural norms they observe in regard to social interactions. Since these interviews aim to collect rich and thick data, the more able interviewers are to detect cultural norms and appropriately respond to these norms, the more likely quality data will be obtained.

This study aims to examine how group interview processes are influenced by cultural norms in a community-based study of Chinese American immigrants, and to examine strategies to facilitate group interview processes vis-a-vis cultural norms. Naturally occurring group processes that emerged during the conduct of interviews were carefully observed. Audio recording of interviews allowed for detailed analysis of group processes and interviewer efforts to facilitate discussion. During data interpretation, we identified linkages between group processes and well known Chinese cultural norms described in the literature. Cultural norms were not pre-selected or manipulated systematically. Employing a postmodern approach (Uba, 2002), we interpreted group interview processes vis-à-vis cultural norms in context.

\section{Method}

\section{Participants}

The sample for the current analysis consisted of 20 foreign-born Chinese American couples, or 40 paired individuals who participated in a qualitative interview study that examined the role of family and culture in type 2 diabetes management (Chesla, Chun, \& Kwan, 2009; Chun, Chesla, \& Kwan, 2011). They were a subsample from a larger comparative study of foreign-born and U.S.-born Chinese Americans living with type 2 diabetes. Participants in 
this analysis had immigrated to the U.S. for 14.71 years on average $(\mathrm{SD}=13.64)$ from either mainland China (11 couples) or Hong Kong. Mean age was 62.29 years $(\mathrm{SD}=9.15)$. All couples were heterosexual and had been married for 33.88 years on average ( $\mathrm{SD}=13.72)$. All spoke Cantonese and lived in the San Francisco Bay Area.

To be eligible, at least one member of a couple had to have diabetes for one year or more. In four of the 20 couples in which both members had diabetes, the person with a longer duration of diagnosis was designated as the patient. Among patients, there were slightly more females (60\%) than males (40\%). Patients' average duration of diagnosis was 8.38 years $(\mathrm{SD}=5.87)$. To treat diabetes, one used diet and exercise only, 17 used oral medications, and two used insulin. Mean hemoglobin A1c was 6.93 ( $\mathrm{SD}=0.96$ ), indicating relatively good glucose control.

Participants were recruited from the San Francisco Bay Area via posting of fliers in public places, referrals from health care providers, community agencies and ESL classes, ethnic media including newspapers, television and radio, and snowballing. They were informed the study aimed to understand how culture and time in the U.S. may influence how Chinese American patients with type 2 diabetes and their spouse or partner understand and care for type 2 diabetes. No diabetes treatment was provided. All completed an informed consent procedure approved by the university IRB. All written study materials used with participants were provided in Chinese only, English only, or in both languages according to participants' preferences.

\section{Design and Procedure}

Overall project-The overall research employed the qualitative research approach of interpretive phenomenology (Benner, 1994; Chan, Brykczynski, Malone, \& Benner, 2010). Interpretive phenomenology aims to understand beliefs and practices in everyday life through a circular process of interpretation. This process involves researchers' preunderstanding about a phenomenon, revelations about the phenomenon from interview data, and researchers' corrected understanding as a result of the revelations. Rather than assuming that an objectively "true" interpretive account exists, this approach considers a better account as one that "goes beyond publicly available understandings of a problem to reveal new and deeper possibilities for understanding" (Leonard, 1994). The rationale for selecting this approach is that it explicitly acknowledges and utilizes researchers' background knowledge about the world and the topic under investigation to guide and facilitate the investigation from data collection through data interpretation. Researchers seek new and deeper understanding of participants' beliefs and practices in relation to the topic in question based on participants' reflections and narratives.

In this project, guiding interview questions and follow-up probes in all interviews were semi-structured and open-ended, and focused on reflections and narratives of diabetes management within the family. For each couple, data collection included six 2-hour interviews, consisting of two couple interviews, two patient group interviews, and two spouse group interviews. A subset of participants $(n=13)$ was interviewed individually if extra time was needed to complete interview questions or if nondisclosure in shared (couple or group) interview settings suggested an individual interview setting would yield more data. Participation in these multiple interviews was scheduled as follows for each couple. Participation began with a couple interview in the home in which rapport was established and the couple was interviewed about their illness and immigration history. A few months after the initial couple interview, the couple was assigned to a group with three to five couples who, like them, were Chinese American immigrants living with diabetes. Patients participated in two patient group interviews, conducted in community service agencies or the university campus, scheduled about a month apart. Spouses participated in two spouse 
group interviews. Group interviews were followed by a second couple interview in the home. Finally, a subset of participants participated in an individual interview in their home. Overall, the multiple interviews in different formats helped reduce reporting of socially desirable data from participants and allowed the research team to triangulate data gathered from different interviews, thus reaching more accurate, richer and deeper interpretation.

All interviews were conducted in Cantonese and audio-recorded digitally. Interviews were translated and transcribed verbatim by a bilingual, bicultural research assistant, and checked by a second bilingual, bicultural research assistant who had conducted the interview for accuracy. Translation discrepancies between original and corrected transcripts were examined by the bilingual, bicultural project director, and the PI and Co-PI of the project when necessary, to decide on the best translation. Final transcripts were imported to the Atlas-ti (Version 5.2) qualitative analyses software for thematic coding, followed by narrative and thematic analyses.

Present analysis-Data for the present analysis came from 20 patient and spouse group interview transcripts. From these transcripts, group processes that arose during discussion were coded "Group dynamics". Coding was done by one member of the research team independently, and cross-checked by the other two members. "Group dynamics" was defined as interpersonal responses to other participants' verbal and non-verbal presentations in a group interview. Next, group processes interpreted to be significantly shaped by published Chinese cultural norms were identified by one member of the team independently, cross-checked by the other two members, and extracted as data for this analysis. Chinese cultural norms were defined as expectations about appropriate and inappropriate behaviors as prescribed by traditional Chinese culture. Group processes interpreted to be shaped by other considerations, such as when patients enthusiastically exchanging diabetes management tips because of a concern for better health, were excluded.

Cross-checking of coding and interpretations of group processes took place during weekly research team meetings and via memo writing in Atlas-ti. Differences were discussed until all three members agreed on the most suitable interpretation. Discussions were guided by each member's own background knowledge about group processes and Chinese cultural norms, the group processes being examined, and each member's holistic understanding of the participants and the group based on all interviews they had completed.

\section{Cultural Adaptations to the Research Process}

Positionality of the research team vis-à-vis participants could have affected participants' engagement in the research. Inclusion of team members from the same culture as those studied at all points in the research process provides insight into cultural processes that might otherwise be overlooked (Skaff, Chesla, Mycue, \& Fisher, 2002). For this study, cross-disciplinary members of varied cultural, immigration and demographic backgrounds comprised the team. Team members who conducted interviews were two bilingual, bicultural, immigrant Chinese American women. Interviewers' background as Chinese immigrants and their capacity to speak the same dialect seemed to enhance participant ease and increase rapport. Other interviewer characteristics, such as younger appearance, higher educational attainment and university research team affiliation, might have led them to be perceived as community outsiders.

However, cultural and demographic positionality of research team did not guarantee engagement with participants. Extra steps were taken to enhance cultural competence of team members through the course of the study. First, great efforts were made to determine appropriate ways to engage participants, such as how to greet participants properly, how to enter their homes showing respect to home rules (e.g. removing shoes), and how to respond 
to special requests (e.g. translation of letters). During data collection, the team regularly reviewed interview fieldnotes, discussed group processes that unfolded in interviews, reflected on interviewers' strategies for handling them, and decided on follow-up actions. Team members brought their varied cultural and life experiences, and diverse training backgrounds and philosophical perspectives, to these dialogues. Second, team members acknowledged their limitations in taking participants' perspectives. They maintained an inquisitive attitude and an open mind in the process of inquiry, and maintained a willingness to make and learn from mistakes when interacting with participants. By embracing each encounter with participants (via actual interviews or transcript reading) as a learning opportunity, over time, the team improved its capacity to adapt the research process to engage and retain diverse Chinese American participants. All twenty couples in this project finished all interviews requested of them, told interviewers that participation had been a positive experience, and agreed to future contact after their participation ended.

\section{Results}

Analysis of group processes in interviews with Chinese American immigrant participants revealed culturally nuanced interaction processes by group members and by interviewers. Significant variability in adherence to cultural norms was apparent, as was the strength of the response by various groups to monitor and enforce the norms. At all times, interviewers strove to monitor and accommodate the appropriate cultural response to the group process, and simultaneously encourage participants' disclosure of personal and family experiences with diabetes. In our interviewing and text interpretation, we attended to participant disclosure at multiple levels. Selection and disclosure of personal reflections and narratives of living with diabetes were a first level of disclosure. Responding to preceding reflections and narratives comprised a second level of disclosure. Interpersonal responses to other participants' verbal and non-verbal presentations in the group comprised a third level of disclosure. In this article, we focus on analyzing the third level of disclosure in the context of the other two.

Group processes were observed to be most prominently influenced by four Chinese cultural norms: sensitivity to social hierarchy, monitoring public display of strong emotions, face concerns, and emphasis on group harmony. These norms were selected because they have been well documented in the literature, and in this study they powerfully shaped group processes, as evidenced in fieldnotes and group interview transcripts. For each norm, we first describe its conventional presentations in published scholarly materials, which encompass Asian and Asian American psychology, communication studies and nursing literatures. We next examine displays of the norm and the manner in which it influenced interview processes, using selected illustrative examples. Finally, we present our practical and inventive responses to these cultural negotiations.

\section{Sensitivity to Social Hierarchy}

According to the literature, Chinese Americans tend to perceive and structure interpersonal relationships in a hierarchical manner (D. W. Sue \& Sue, 2003). Depending on the presence of others and their perceived positions in the social hierarchy (e.g. class, age, gender, family role), some Chinese Americans may adjust what they reveal and how they present themselves. Such high-context ways of presenting oneself and relating to others are rooted in the Confucian prescription that individuals should understand their proper relational roles and fulfill the corresponding duties to achieve societal peace and harmony (Park \& Chesla, 2007).

Social hierarchy as perceived by participants strongly affected their interactions in group interviews. Participants' disclosure patterns and turn-taking frequently depended on the 
perceived power and status of other group members. Members deferred to those who appeared to be of high socioeconomic, educational, or professional status, and great acculturation level. Deference to older participants was less apparent, but also noted. These dynamics were illustrated in an interview with a group comprised of a woman of high socioeconomic status (SES) who had resided in the U.S. for a few decades (Mrs. A), a woman of much lower SES who had resided in the U.S. for only a few months (Mrs. B), and a middle-class man with 10 years in the U.S. (Mr. C). In the first group interview, Mrs. A's social class was apparent as she discussed her family's thriving business and abundant resources, including family maids and a chef. In addition, Mrs. A wore fine-quality clothes and large jewelry while the others wore modest street clothes. In the beginning of the second group interview, participants were asked for a story of when they had been able to support their spouses in managing diabetes. Mrs. B demurred.

Mrs. B: What do we talk about? I don't understand. [Chuckles from speaker and others]

Should we talk about things that are good and bad, these kinds of things?

The interviewer responded by rephrasing the question and providing examples. Mrs. B demurred again and deferred to Mrs. A to speak. Mrs. A agreed that she had nothing to say, but then provided an extended narrative about her husband's diabetes. When she finished speaking, the interviewer asked the others for narratives and $\mathrm{Mr}$. $\mathrm{C}$ took his turn to speak. Mrs. B waited to speak last. Later in the interview, this pattern of deferring to higher SES participants was repeated: Mrs. A spoke first, the middle-class man spoke second, and Mrs. B spoke last.

Shifts in level of disclosure were at times dependent upon hierarchies and upon an individual's choice of narratives. In the same group Mr. C spoke openly about his concern for his wife during hypoglycemic episodes. His disclosure appeared to increase Mrs. B's confidence to speak across hierarchies, since she later directed questions to him and disclosed difficulties. When Mrs. B narrated about her husband's diabetes, she openly disclosed doubts about her husband's interpretation of diabetes. She noted her husband's alleged lack of strength and expressed skepticism about his symptom. She asked other participants repeatedly if they knew whether diabetes would deplete a person of strength. Her active questioning suggests that, despite initial sensitivity to social hierarchy, she grew comfortable with the group and eventually used it to gather information. At the same time, she was more inhibited in group interviews than in subsequent one-on-one interview, in which she frankly discussed couple disagreements.

Many participants exhibited sensitivity to social hierarchy by deferring to participants of higher perceived status. Those of higher perceived status diffused power differentials by their willingness to relate to others, via sharing experiences and offering suggestions. Interviewers accommodated hierarchical norms and diffused power differentials simultaneously by encouraging participants to speak freely, making eye contact and maintaining rapport with all participants, and managing time to prevent anyone from dominating the discussion. Additionally, interviewers paced interviews according to participants' level of comfort. Although all were asked to speak, interviewers made clear participants controlled when and how they contributed.

Norms regarding social hierarchy affected how participants interacted with interviewers, who were often perceived as authority figures or sources of expert knowledge about diabetes and health. Researchers explained their roles during screening and informed consent, yet were commonly perceived as medical experts. This happened most often with the first author, who used her professional title as a Doctor of Philosophy, which was misunderstood 
to mean Medical Doctor in Chinese. Such misperceptions led to positive and negative reactions. Some participants cajoled interviewers to share their expertise regarding diabetes.

Mrs. E: You are physicians, you should know. You should instruct us.

When participants realized that the interviewers could not impart medical advice, it sometimes raised concerns about interviewers' capacity in understanding their experiences.

Mrs. E: What is your own knowledge of diabetes? How much understanding do you have?

When interviewers responded that they had a general understanding of diabetes but were not medical providers and could not give medical advice, most seemed satisfied.

Other participants, assuming interviewers adhered to western biomedical philosophies, questioned interviewers' openness to their perspectives.

Mr. F: Perhaps Dr. X doesn't believe in traditional Chinese medicine too much.

Mrs. G: Rare folk formulas ( 民間獂方). [Chuckle]

Mr. F's comment may have embarrassed or amused other participants because it challenged the authority of the interviewer and her capacity to do her job. Observing the hierarchy and smoothing the discussion, Mrs. G described the Chinese medicine treatment under discussion using a term that highlighted its eccentricity and dubiousness. In response, the interviewer disagreed with Mr. F's comment in a friendly tone of voice and emphasized the value of participants' practical knowledge and belief in all forms of treatment.

One patient perceived the interviewers to be "government" representatives, possibly because of their public university affiliation and the federal research funding. He thanked the government via the interviewers on several occasions.

Mr. D: [University] is attaching so much importance to us patients with diabetes, specifically organizing several interviews .... We as patients would like to express our thanks to the government and the related units.

It is likely that Mr. D's presentations in the study were constrained by his construction of interviewers as governmental representatives. Emigrating from a country where government censorship was possible, he likely modified his disclosures for personal safety. Efforts to correct his view of the project's relationship with governmental authority were continuous, but not successful in dispelling his doubt.

\section{Monitoring Public Display of Strong Emotions}

An open display of strong emotions is considered by some Chinese Americans to be a sign of character weakness or immaturity (D. W. Sue \& Sue, 2003). Lack of moderation in expressing emotions is undesirable because emotions may disrupt social harmony and status hierarchy (Bond, 1993). On the other hand, "Hanxu" ( 含第), a mode of verbal and nonverbal communication that is contained, reserved, implicit and indirect, is often considered desirable and appropriate. "Hanxu" entails monitoring overt emotional expressions, especially strong and negative emotions (Gao, Ting-Toomey, \& Gudykunst, 1996).

Concerns over public display of strong emotions affected participants' behaviors and disclosure in many group interviews. When strong emotions were expressed, participants reacted with social cues and statements that encouraged moderation, including dampening or diverting from emotionally difficult topics. Less often, groups accommodated and empathized with the speaker's powerfully expressed feelings of disappointment or sadness. For example, when one participant cried, others helped him regain composure. 
Mr. I: Actually, after my wife found out about diabetes, the first effect was on her mood. [9 second pause. He started to cry.] I am sorry, I [couldn't continue].

The interviewer and other participants immediately offered empathy and support, and acknowledged the difficulty of Mr. I's experience. Mrs. J then asked a factual question to explore the cause of his wife's diabetes in a light-hearted tone, thus attempting to divert Mr. I from his intense sadness. Mr. I tried to continue but sniffled and paused again. The interviewer again acknowledged the difficulty of the experience and normalized it. Another participant, Mr. K, then spoke up. The interviewer took the opportunity to direct the conversation to Mr. K, who then talked about how he encouraged his wife to cope with diabetes.

Mr. I's break in composure affected the entire group tremendously. The interviewer gently offered the possibility for him to continue or stop speaking, normalized his emotion, and expressed empathy. Group members were attentive, and Mr. K provided diversion. Mr. I got up to get bottles of water and regained composure. As the interview continued, Mr. I did not comment further on his wife. Other participants and the interviewer did not question him further.

After the interview, Mr. I approached the interviewers in private, apologized for what happened and almost began to cry again. When interviewers tried to comfort and reassure him that it was normal to express sadness and cry, he said, "But I am a man, so I shouldn't [cry]". His pointed apology suggested he believed he had violated a norm and behaved inappropriately. Efforts to reassure him were apparently successful as he returned to subsequent groups, completed the study and offered to serve as a volunteer for the study in the future.

Groups frequently tried to help participants return to a state of composure and avoid emotional topics, reflecting a collective belief that crying or strong displays of emotions were not desirable in public. These incidents challenge the stereotype that Chinese Americans, especially males, might be stoic or lacking in strong emotions. It is likely that when individuals experience strong emotions in public, they self-monitor or respond to social cues, and therefore do not express those emotions fully. When participants expressed intense emotions, interviewers accommodated the norm of moderation in emotional display and at the same time, allowed participants to express emotions as they felt comfortable. Unmonitored emotional expression by one participant might make other participants uncomfortable even if they felt empathic. On the other hand, honest reflection on and narration of experiences with chronic illness often elicited feelings of grief or loss. In addition, a group interview context may have provided opportunities for emotional expressions that were not available within the family. To balance all priorities, interviewers normalized distress, offered comforting remarks, provided time for participants to regain composure, and provided opportunities for others to comfort or distract the participant.

\section{Face Concerns}

Face concerns are considered characteristic of Chinese and other Confucian-based cultures. According to Ho (Ho, 1976), "in terms of two interacting parties, face is the reciprocated compliance, respect, and/or deference that each party expects from, and extends to, the other party." In social interactions, some Chinese may attend to gaining face for oneself, preventing loss of face for oneself, giving face to others, and preventing loss of face for others. Being able to understand, practice, and balance these face concerns in interpersonal encounters are central in the development and regulation of social relationships among Chinese (Chang \& Holt, 1994). Furthermore, a person's face can affect the face of the family, as individuals are seen as representative of and inseparable from their families. In 
Asian American psychology, face has been defined as "a person's set of socially-sanctioned claims concerning one's social character and social integrity in which this set of claims or this 'line' is largely defined by certain prescribed roles that one carries out as a member and representative of a group" (Zane \& Yeh, 2002). Face concerns for oneself and one's family might explain Asian Americans' high premature termination rates and short treatment stays in mental health systems. If a therapist encourages clients' disclosure without attending to face concerns, the resulting loss of face could be counter-therapeutic and highly distressing (Zane \& Mak, 2003).

Interviewers relied on careful observations of group members' behavior to identify moments when face concerns affected group processes. When participants withheld negative information and provided socially desirable information, fear of losing face and/or an interest in gaining face for oneself might have been the motivation. Observing the same norm, other group members, on realizing presentations that were unduly positive or untrue, might prevent a loss of face for others or try to save face for others by avoiding exposure of the true state of affairs even when it had become evident. Interviews with couples outside of group interviews sometimes contradicted participants' disclosure in groups. Such information provided clues to moments when face concerns might be in play. The following example illustrates these dynamics.

In a first spouse group interview, one husband (Mr. L) suggested that his wife maintained good control of her diabetes, did not need to take diabetes medications, and attributed this success to his effective coaching. He additionally denied diabetes-related couple difficulties. However, the interviewers knew from a prior interview that Mrs. L took diabetes medications. Additionally, in patient group interviews conducted separately from spouses, Mrs. L discussed Mr. L's attempts to dissuade her from taking her diabetes medications. Mr. L's statements in the spouse group interview were likely an effort to gain face. Subsequent exchanges supported this interpretation. As the interview progressed, other spouses complimented Mr. L for his successful management of his wife's diabetes, marked by the fact that she did not need medications. Mr. L listened to their conversation in silence, gaining face as others complimented him. However, Mr. L subsequently revealed a limited understanding of diabetes and unknowingly contradicted his earlier presentations. He told a story about his wife testing her glucose using a glucose meter.

Mr. L: What measurement is considered high? ... I have tried buying one from a pharmacy for $\$ 9.99$ to stick our fingers. My wife pressed on the device once, it was already about an hour after she ate, it went up to between 200 and 210. She said, "It doesn't make sense. How come it is still so high more than an hour after I ate?"

Mrs. M: You may only check two hours after you eat.

Mr. L: So then the next morning, we tested again. "It's 200 something again?", she said.

When the interviewer asked Mr. L whether he asked the doctor about these readings, he said he had not and had thrown away his wife's meter. The group's atmosphere became awkward. From conversing in a lively manner, other spouses looked surprised and became quiet. Perhaps realizing the implications of what he had revealed, Mr. L looked uneasy. After a pause, other spouses described normal glucose levels. No one confronted Mr. L about his discrepant presentations, thereby preventing him from losing face. In turn, Mr. L seemed to gradually develop greater trust in the group and disclosed more honestly. For the first time he admitted to having diabetes-related couple conflicts.

Throughout group interviews, it was apparent that participants were aware and respectful of face concerns. This contributed to greater group rapport and more honest disclosure from 
those who feared losing face. Similarly, interviewers showed sensitivity to participants' face concerns by reminding participants of their right to speak or not speak to any particular question.

Interviewers did not confront even when it was obvious that participants withheld or misrepresented information. Did attending to face concerns prevent the research team from learning the "truth" about illness and family experiences? It is likely that some participant responses were face presentations rather than honest disclosures. Collecting data in multiple interviews over time, and in multiple interpersonal contexts, including the possibility of a one-on-one interview, allowed for trust building and disclosure of personal concerns out of the presence of family members. In addition, we observed that because of interviewers' expressed sensitivity to face concerns, participants often engaged with the research process and became more disclosing of positive and negative aspects of their experiences. Almost all participants, even the ones who showed great face concerns initially, eventually disclosed some difficulties.

\section{Emphasis on Group Harmony}

Group harmony is highly desirable in Chinese culture. In general, Chinese individuals seek to maintain good social relationships and to avoid direct confrontations (Gao et al., 1996). When social harmony is jeopardized, social relations may be adversely affected, thus constituting a potentially serious threat to Chinese individuals who tend to have an interdependent sense of selves (Markus \& Kitayama, 1991) and a collectivist social orientation (Triandis, 1995).

Consistent with this norm, group interviews with Chinese immigrant participants were generally harmonious and interpersonal exchanges were pleasant and respectful. When differences of opinion were expressed, participants frequently employed resolution strategies that promoted group harmony and minimized conflicts. In scores of hours of group interviews, no participant challenged other group members in a strong or aggressive manner.

The following exchange illustrates how skillfully yet subtly group members enacted a norm of group harmony. The exchange began when a wife, Mrs. O, expressed doubt that her husband's low energy was a symptom of diabetes. Objecting, another wife, Mrs. P, coached her to trust her husband. In response, Mrs. O diverted to an easier topic, the possible causes for her husband's alleged lack of energy, rather than the more personal issue of whether she should believe in her husband. She did not respond to Mrs. P's advice directly.

Mrs. P: Do not refuse to believe him. I heard my husband said that he too lost energy.

Mrs. O: I don't know whether it is the medication or the fact that he has not eaten rice.

As this exchange proceeded, Mrs. P stopped her coaching and talked about diabetes medications as a possible cause for fatigue. The interviewer then commented on the diverse factors that could affect a patient's strength. By not responding to the relationship advice directly, Mrs. O minimized differences in opinions and thus promoted group harmony. Similarly, by desisting from her coaching, Mrs. P minimized the possibility of direct confrontation. Having sensed that the norm of group harmony was operating, the interviewer acknowledged the diverse possibilities underlying fatigue and did not encourage the women to explicate their positions. 
Preservation of group harmony was also possible in the face of opposite opinions. Careful encouragement by the interviewer and respectful presentations by participants allowed divergent opinions to be expressed. In one such exchange Mr. R praised and thanked the U.S. government for the excellent healthcare assistance it provided to new immigrants. Mr. Q looked distressed.

Mr. Q: I'd like to speak. But my situation is just the opposite of this Mr. R's.... I haven't received any benefits from the U.S. government.

Mr. Q talked at length about his family's financial hardships and his ineligibility for governmental assistance. His expressed distress at listening to Mr. R suggested that his experiences were deeply felt. Despite this, he described his experience with composure and did not challenge Mr. R, thus preserving group harmony. Group members were initially surprised, but subsequently expressed empathy for Mr. Q's financial predicament. Contrary perspectives were validated and clarified by interviewers to encourage their continued expression.

Concerns for group harmony sometimes dampened discussions of difference. By adopting a tone of neutrality and curiosity, interviewers were able to create an opening for the divergence to reappear. An example arose when three patients were asked about difficulties in seeking health care. Two patients suggested they had "no difficulties" before a third identified the problem of not understanding English. The two patients reacted to her comment by speaking extensively about the role of interpreters in helping them access health care easily. Eventually, the third patient said, "there aren't any difficulties". Concerns for group harmony likely explain the change in her response. The interviewer then attended neutrally to all viewpoints, and encouraged each member to expand on their statements. Eventually, the third patient elaborated her experiences with language difficulties and the other two disclosed similar experiences.

Although concerns for group harmony prevailed, some participants' non-verbal behaviors challenged harmony. For example, during a group interview when a participant talked about her husband's diabetes, a male took out his newspapers and pointed out a headline to another male, engaging him in a brief conversation. He then went on to read his newspapers, ignoring the speaker, a soft-spoken older woman dressed in heavily-worn clothing. The male participant likely ignored her comments because he perceived her to be of lower status. In other instances, participants engaged in side conversations while group members were narrating. These behaviors could have been perceived as disrespectful to the primary speaker, thus undermining rapport and even leading to conflicts. Interviewers intervened when such behaviors became disruptive to the interview process. Perhaps observing the norm of harmony, none of the participants who were ignored protested. Also, when interviewers intervened, participants who behaved disrespectfully always complied with interviewers' suggestions.

In all, participants sought to maintain group harmony in the face of different or opposite opinions. Interviewers accommodated this norm and encouraged disclosure of divergent perspectives simultaneously by highlighting unique situations in different families, encouraging participants to discuss their own experience, and normalizing differences of opinion. Harmony concerns could be counter-productive when they dampened divergent discussions. When that happened, interviewers coped by attending to nuances in discussions and probing minority viewpoints, so as to promote discussion of diverse perspectives. Lastly, challenging this norm, some participants enacted behaviors that communicated disrespect to others. However, perhaps due to the overpowering impact of this norm, no protests or conflicts resulted. 


\section{Discussion}

This article provides practical illustrations of how group dynamics in group interviews with Chinese American immigrants were shaped by cultural norms regarding sensitivity to social hierarchy, monitoring public display of strong emotions, face concerns, and emphasis on group harmony. Confirming guidelines on research methods with Asian Americans, we found it feasible to use group interviews to collect data from Chinese American immigrants. Enriching these guidelines, this article presents a data-based examination of group processes in research group interviews. It reveals how group processes were shaped by cultural norms and how interviewers' responses to group processes ensured group rapport and participant disclosure.

While we highlight group processes with significant cultural underpinnings, we do not intend to reinforce stereotypes. In fact, we noted variability in group processes that diverged from cultural norms, such as disharmonious inattention or talking over a primary speaker. Rather than invalidating cultural norms, counter-examples underscore their complexity. They alert researchers to be observant and keep an open mind to variations in participants' adherence to cultural norms, while striving to be culturally-sensitive. Methodologically, the unplanned, unrehearsed exchanges in group interviews allowed these variations to emerge, thereby yielding data that shed light on the complexity of cultural norms.

Group processes that arose naturally in these qualitative interviews reflect the intersectionality of participants' multiple identities. Participants' multiple identities (as a Chinese American immigrant and as a participant, a man or a woman, a person of higher or lower SES, a patient or a spouse, etc.) affected the ways in which cultural norms asserted their influence. In data analyses, we examined how participants tended to, but did not always, adhere to cultural norms in their own ways and how interviewers negotiated cultural norms in their own ways, given their multiple identities (as Chinese American immigrant female interviewers). As such, this inquiry is distinct from studies that assume an essentialized view of culture; it presents data-based complications to prescriptive guidelines on working with Chinese Americans.

Additional factors bear consideration when adapting to cultural norms in group interviews with Chinese American immigrants. They include individual differences within cultural groups as a function of other factors such as gender, age, SES, and acculturation, which affects the degree to which participants understand and adhere to Chinese norms versus other norms (Chun \& Akutsu, 2003). Researchers must constantly examine pre-conceptions against observable facts and behaviors. Research will be strengthened by "scientific mindedness" (S. Sue, 1998), forming and testing cultural hypotheses in the course of data collection, given the contextualized, multi-dimensional and time-evolving nature of culture and cultural norms.

Relationships among multiple cultural norms require clarification. In this article, group processes were discussed in relation to a single norm for the sake of clarity. In reality, multiple norms operate simultaneously. For example, a participant deferring to another participant of higher SES demonstrates sensitivity to social hierarchy and face-giving, both of which contribute to group harmony. Similarly, avoiding direct confrontations about another participant's discrepant self-presentations shows an effort to prevent loss of face and requires moderation in public display of strong emotions (e.g. by not looking shocked). In actual interviews, it is important to attune to the concurrent working of multiple norms. In addition, not all norms that affect group processes were examined. For example, the norm of modesty was observed, such as when participants claimed lack of knowledge about diabetes but provided narratives that illustrated their good diabetes knowledge and skills. Modesty 
affected group processes less frequently and powerfully than the four norms under focus and thus was not explored. Interviewers still had to attune to these less prominent norms as necessary.

This study had strengths and limitations. We argue that current findings are specific to Chinese American immigrant culture. However, as in most cultural or indigenous studies, without comparable cross-cultural data, we are unable to definitively conclude that these findings are culturally unique. To address this question, cross-cultural studies that compare group processes in Chinese American immigrant versus other cultural groups are needed.

There may be alternative explanations (e.g. gender, age, personal habits) for the observed group processes other than cultural norms. During interpretation, we evaluated alternative explanations and searched for disconfirming evidence to our interpretations. We included as study findings only group processes that all team members agreed to be best explained by particular Chinese cultural norms, based on our background knowledge of group processes and Chinese cultural norms, as well as our holistic understanding of the participants from multiple interviews in different settings. We suggest that our cultural norm interpretation is reasonable, given our understanding of the whole of participants' behaviors and utterances. However, since all interpretations, even those carefully derived from rich interview text, are tentative and partial (Smith, Flowers, \& Larkin, 2009), we cannot claim with complete certainty that cultural norms are solely responsible for group interview processes. Interviewers might offer verbal probes or interpretations about group processes within the interviews to confirm, or disconfirm, that our read on participant behaviors is apt; however, such interpretations on the spot might change participant behaviors and interfere with naturally occurring group processes.

Findings from this study may not generalize to non-Cantonese speaking Chinese Americans or Chinese Americans born in the U.S., because they may operate under cultural norms different from the ones prominent among Cantonese-speaking foreign-born Chinese Americans. Findings also may not generalize to other Asian American ethnic groups because of each group's unique cultural characteristics. Nonetheless, current findings have high contextual validity because most, if not all, relevant features of group interviews with Chinese American immigrants were taken into account in data collection and interpretation (Marecek, 2003). At a broader level, current findings underscore the importance of attending to group dynamics in interviews as shaped by norms of a particular cultural group, and accommodating or diffusing cultural norms as needed to facilitate interviews. This lesson can sensitize researchers who conduct group interviews with diverse Asian American groups (Marecek, 2003). Researchers may be more able to collect quality group interview data by familiarizing themselves with the literature on cultural norms for the study population, being flexible when encountering group processes that appear to be affected by cultural norms, and in shifting interview practices to accommodate or diffuse those norms. As demonstrated here, the interviews themselves can be instructive about current practices in handling dynamic and evolving cultures and cultural norms.

Lastly, because all participants were patients or spouses living with diabetes, findings may not generalize to people who do not live with diabetes. On the other hand, although having diabetes complicates patients' and spouses' lives with disease management requirements such as taking medications and glucose monitoring, other management activities parallel general health requirements, such as managing diet and exercise. No evidence suggests living with diabetes changes participation in group discussions vis-a-vis cultural norms.

The data-based lessons sensitize and inform researchers to the complex role of cultural norms in shaping group processes in interviews with Chinese American immigrants. As 
Asian American psychology strives to expand and enrich its understanding of Asian American experiences, these lessons can help enhance cultural competence of researchers and enable collection of quality group interview data.

\section{Supplementary Material}

Refer to Web version on PubMed Central for supplementary material.

\section{References}

Benner, PE. Interpretive Phenomenology: Embodiment, Caring, and Ethics in Health and Illness. Thousand Oaks, CA: Sage Publications; 1994.

Bond MH. Emotions and their expression in Chinese culture. Journal of Nonverbal Behavior. 1993; 17(4):245-262.

Chan, GK.; Brykczynski, KA.; Malone, RE.; Benner, P. Interpretive Phenomenology in Health Care Research. Indianapolis, IN: Sigma Theta Tau International; 2010.

Chang, H-C.; Holt, RG. A Chinese perspective on face as inter-relational concern. In: Ting Toomey, S., editor. The challenge of facework: Cross-cultural and interpersonal issues. Albany, NY: State University of New York Press; 1994. p. 95-132.

Chen H, Kramer EJ, Chen T, Chung H. Engaging Asian Americans for Mental Health Research: Challenges and Solutions. Journal of Immigrant Health. 2005; 7(2):109-116. doi:10.1007/ s10903-005-2644-6. [PubMed: 15789163]

Chesla C, Chun KM, Kwan CM. Cultural and family challenges to managing type 2 diabetes in immigrant Chinese Americans. Diabetes Care. 2009; 32(10):1812-1816. doi: 1810.2337/ dc1809-0278. [PubMed: 19628812]

Chun, KM.; Akutsu, PD. Acculturation among ethnic minority families. In: Chun, KM.; Organista, PB.; Marin, G., editors. Acculturation: Advances in theory, measurement, and applied research. Washington, DC: American Psychological Association; 2003. p. 95-119.doi:10.1037/10472-008

Chun KM, Chesla CA, Kwan CML. "So We Adapt Step by Step": Acculturation experiences affecting diabetes management and perceived health for Chinese American immigrants. Social Science \& Medicine. 2011; 72(2):256-264. doi: 210.1016/j.socscimed.2010.1011.1010. [PubMed: 21147509]

Chun, KM.; Morera, OF.; Andal, JD.; Skewes, MC. Conducting research with diverse Asian American groups. In: Leong, FTL.; Inman, A.; Ebreo, A.; Yang, L.; Kinoshita, L.; Fu, M., editors. Handbook of Asian American psychology. 2nd. Thousand Oaks, CA: Sage Publications; 2007. p. 47-65.

Gabrenya, WK., Jr; Hwang, K-K. Chinese social interaction: Harmony and hierarchy on the good earth. In: Bond, MH., editor. The handbook of Chinese psychology. New York, NY: Oxford University Press; 1996. p. 309-321.

Gao, G.; Ting-Toomey, S.; Gudykunst, WB. Chinese communication processes. In: Bond, MH., editor. The handbook of Chinese psychology. New York, NY: Oxford University Press; 1996. p. 280-293.

Ho DYF. Concept of Face. American Journal of Sociology. 1976; 81(4):867-884.

Iwamasa, GY.; Sorocco, KH. Aging and Asian Americans: Developing culturally appropriate research methodology. In: Nagayama Hall, G.; Okazaki, S., editors. Asian American psychology: The science of lives in context. Washington, DC: American Psychological Association; 2002. p. 105-130.

Lee, E. Working with Asian Americans: A guide for clinicians. New York, NY: Guilford; 1997.

Lee, E.; Mock, MR. Chinese Families. In: McGoldrick, M.; Giordano, J.; Garcia-Preto, N., editors. Ethnicity and family therapy. 3. New York, NY: Guilford Press; 2005. p. 302-318.

Leonard, VW. A Heideggerian phenomenological perspective on the concept of a person. In: Benner, P., editor. Interpretive phenomenology: Embodiment, caring, and ethics in health and illness. Thousand Oaks, CA: Sage; 1994. p. 43-63.

Leung, K. The role of beliefs in Chinese culture. In: Bond, MH., editor. The handbook of Chinese psychology. New York, NY: Oxford University Press; 1996. p. 247-262. 
Marecek, J. Dancing through minefields: Toward a qualitative stance in psychology. In: Camic, PM.; Rhodes, JE.; Yardley, L., editors. Qualitative research in psychology: Expanding perspectives in methodology and design. Washington, DC: American Psychological Association; 2003. p. 49-69.

Markus HR, Kitayama S. Culture and the self: Implications for cognition, emotion, and motivation. Psychological Review. 1991; 98(2):224-253. doi: 210.1037/0033-1295X.1098.1032.1224.

McGoldrick, M.; Giordano, J.; Garcia-Preto, N., editors. Ethnicity and family therapy. 3. New York, NY: Guilford Press; 2005.

Okazaki, S.; Lee, RM.; Sue, S. Theoretical and conceptual models: Toward Asian Americanist psychology. In: Leong, FTL.; Inman, A.; Ebreo, A.; Yang, L.; Kinoshita, L.; Fu, M., editors. Handbook of Asian American psychology. 2. Thousand Oaks, CA: Sage; 2007. p. 29-46.

Park M, Chesla C. Revisiting Confucianism as a conceptual framework for Asian family study. Journal of Family Nursing. 2007; 13(3):293-311. doi: 210.1177/1074840707304400. [PubMed: 17641110]

Skaff MM, Chesla CA, Mycue VdlS, Fisher L. Lessons in cultural competence: Adapting research methodology for Latino participants. Journal of Community Psychology. 2002; 30(3):305-323. doi: 310.1002/jcop.10007.

Smith, JA.; Flowers, P.; Larkin, M. Interpretative phenomenological analysis. London, England: Sage; 2009.

Sue, DW.; Sue, D. Counseling the culturally diverse: Theory and practice. 4. New York, NY: John Wiley \& Sons; 2003.

Sue S. In search of cultural competence in psychotherapy and counseling. American Psychologist. 1998; 53(4):440-448. doi: 410.1037/0003-1066X.1053.1034.1440. [PubMed: 9572007]

Triandis, HC. Individualism \& Collectivism. Boulder, CO: Westview Press; 1995.

U.S. Census Bureau. Population Change and Distribution 1990 to 2000. Census 2000 brief. 2001. Retrieved from http://www.census.gov/prod/2001pubs/c2kbr01-2.pdf

Uba, L. A postmodern psychology of Asian Americans: Creating knowledge of a racial minority. Vol. 202. Albany, NY: State University of New York Press; 2002.

Zane, N.; Mak, W. Major approaches to the measurement of acculturation among ethnic minority populations: A content analysis and an alternative empirical strategy. In: Chun, KM.; Organista, PB.; Marin, G., editors. Acculturation: Advances in theory, measurement, and applied research. Washington, DC: American Psychological Association; 2003. p. 39-60.doi:10.1037/10472-005

Zane, N.; Yeh, M. The use of culturally-based variables in assessment: Studies on loss of face. In: Kurasaki, KS.; Okazaki, S.; Sue, S., editors. Asian American mental health: Assessment theories and methods. New York, NY: Kluwer Academic/Plenum Publishers; 2002. p. 123-138. 